\title{
Linear stability of a horizontal phase boundary subjected to shear motion
}

\author{
A. Kheniene and A. Vorobev \\ Faculty of Engineering and the Environment, University of Southampton, UK
}

Received: date / Revised version: date

\begin{abstract}
We investigate the stability of slowly smearing phase boundary that appears at the contact of two miscible liquids. A hydrodynamic flow is imposed along the boundary. The aim is to find out whether the slow diffusive smearing of a boundary can be overrun by faster mixing. The phase-field approach is used to model the evolution of the binary mixture. The linear stability in respect to $2 D$ perturbations is studied. If the heavier liquid lies above the lighter liquid, the interface is unconditionally unstable due to the Rayleigh-Taylor and Kelvin-Helmholtz instabilities. The imposed flow accelerates the growth of the long-wave modes and suppresses the growth of the short-wave perturbations. Viscosity, diffusivity and capillarity reduce the growth of perturbations. If the heavier liquid underlies the lighter one, the interface can be stable. The stability boundaries are defined by the strength of gravity (density contrast) and the intensity of the imposed flow. Thinner interfaces are usually characterised by larger zones of instability. The thermodynamic instability, identified for the thicker interfaces with the thicknesses greater than the thickness of a thermodynamically equilibrium phase boundary, makes such interfaces unconditionally unstable. The zones of instability are enlarged by diffusive and capillary terms. Viscosity plays its stabilising role.
\end{abstract}

PACS. 47.20.-k Flow instabilities - 47.20.Ft Instability of shear flows - 68.05.-n Liquid-liquid interfaces - 64.70.Ja Liquid-liquid transitions

\section{Introduction}

We investigate the dynamics of mixing initiated by a contact of two miscible liquids. The miscible liquids are represented by the components of an off-equilibrium binary mixture. The process mixing involves hydrodynamic flows near the phase boundary, but, ultimately, the the equilibration of the binary mixture occurs though the interfacial diffusion.

The mixing rate is obviously increased by enlargement of the interfacial contact area due to disintegration of the phase boundary. For instance, the classical RayleighTaylor instability develops if the lighter liquid underlies the heavier one. The linear stability of such an interface was studied in ref. [1], where it was shown that the interfacial diffusion slows down the development of hydrodynamic modes. From the other hand, in [1], it was also shown that thick interfaces, with the thicknesses exceeding the thickness of a thermodynamically equilibrium phase boundary, are prone to the new thermodynamic instability. Thus, the thick interface would be thermodynamically unstable, and development of the instability would inevitably be accompanied by hydrodynamic motion, even if the lighter liquid lies over the heavier liquid. In the present work, the results of ref. [1] are extended by examining the effects introduced by a shearing motion imposed along the phase boundary.

Understanding of the mixing dynamics is obviously important for accurate modelling of various natural phenomena, such as atmospheric and ocean mixing, and for modelling of various processes in chemical engineering that involve the mixing of chemicals, and also for modelling such processes as the oil recovery [2], extraction of essential oils [3], etc. In addition, there is a growing interest to model the flows in micro-cavities (micro chemical reactors), where the mixing between different substances is sometimes desirable but is limited due to suppression of hydrodynamic instabilities [4]. A recent review on peculiarities of the mixing dynamics of two different liquids can be found in ref. [5].

A shear flow in a homogeneous liquid is known to be unconditionally unstable, with the most unstable perturbations having the form of $2 D$ billows with the axes perpendicular to the streamwise direction. This is known as the Kelvin-Helmholtz instability [6,7]. The situation becomes more complicated if the flow is imposed along the interface separating two liquids of different densities. In the case of gravitationally unstable configuration (the heavier liquid superposes the lighter liquid) the RayleighTaylor and Kelvin-Helmholtz instabilities co-develop. These two instabilities primarily affect the modes of different 
wavelengths, so they do not amplify each other, and, on opposite, the imposed flow may even reduce the growth of the modes with shorter wavelengths [8-10]. The codevelopment of these instabilities was already studied for smeared interfaces $[11,12]$. In particular, it was found that interface smearing results in lower growth rates of the short length modes, and in increased growth rates of the modes with longer wavelengths.

A gravitationally stable configuration, with the heavier liquid underlying the lighter one, may be stable or unstable, with the stability defined by the Richardson number, that is introduced as the ratio between the gravitational and kinetic energies. If the external flow is non-existent, disturbances of an interface induce gravity-capillary waves that decay due to viscous damping. The external shear flow of sufficiently large amplitude makes the interface unstable through the Kelvin-Helmholtz and Holmboe instabilities $[6,13,7]$. The growth of perturbations due to the Kelvin-Helmholtz instability occurs monotonically with zero phase speed. The Holmboe instability occurs in a stratified layer through the growth of two travelling modes (the modes with non-zero phase speeds). The boundaries of the Kelvin-Helmholtz and Holmboe instabilities were later determined for the various density and velocity profiles [14-19], including the non-symmetric profiles with non-coincident central levels of the basic profiles of density and velocity [20,21]. The effect of viscosity and diffusivity on the stability of stably stratified shear flows was studied in ref. [22], where it was found that there is no simple rule to hold about whether viscosity and diffusivity stabilize or destabilize the flow. The effects of surface tension and larger density contrasts were also studied in refs. $[23,24]$. Finally, the existence of both Kelvin-Helmholtz and Holmboe instabilities were experimentally confirmed in e.g. refs. [25-27]. The further non-linear stages of the shear flow instability were examined in e.g. refs. [28,29].

In the current work we investigate the stability of a phase boundary that separates two components of an isother-a mal binary mixture. The binary mixture is initially in a state that is different from the state of thermodynamic equilibrium. The thermo- and hydrodynamic evolution of such a mixture is defined by the full Cahn-Hilliard-NavierStokes equations [30], which set the so-called quasi-compressible system of equations due to dependence of the mixture density on concentration. The slow convective and diffusive evolution of liquid/liquid systems is defined by the reduced Boussinesq approximation [31]. The main difference of the current work from the above-cited studies, is that the stability of a phase boundary is investigated for the multiphase system with fully defined thermodynamics. In other above-cited works, the instability of a smeared density profile (a stratified layer) was studied, with the diffusion defined by the classical Fick's law.

We investigate the influence of the shear flow imposed along the horizontal interface on the interface stability, assuming that the inflection points for both density and velocity profiles coincide.

The paper is organised as follows. The mathematical model is defined in sect. 2 . In the same sect. we develop the
$2 D$ linear stability theory for the introduced basic profiles of density and velocity. In the next two sects. 3 and 4 , we discuss the stability results. In sect. 3 , the stability of a smeared interface between two immiscible liquids is examined, i.e. in this sect. we assume infinite value for Peclet number. In sect. 4 , the role of diffusivity on the stability is investigated. Both sects. 3 and 4 contain the discussions of the instabilities developing in the gravitationally-stable and gravitationally-unstable configurations. The conclusions are summarised in sect. 5 .

\section{Mathematical model}

\subsubsection{Governing equation}

When pure components of a binary mixture are brought into contact, the molecules of the neighbouring liquids inter-diffuse, so the binary mixture changes its state to thermodynamic equilibrium. Typically this transition is very slow (takes hours). The final equilibrium states of a mixture are usually defined by a phase diagram [32]. We focus on the binary mixtures with the upper critical solution temperature. For such a mixture, the equilibrium state can be heterogeneous or homogeneous (dependent on the mean concentration) if the mixture temperature is below the critical value. And the mixture is always homogeneous in equilibrium at the temperatures above the critical point. Some binary systems has no critical point, and they are miscible under all temperatures, e.g. water and glycerol or hexane and soybean oil [33]. We presume that the behaviour of such mixtures is similar to the behaviour of the mixtures with the upper critical solution temperatures in the supercritical conditions.

The evolution of the heterogeneous binary mixture is modelled on the basis of the phase-field approach. This approach was first suggested in the works of van der Waals and Cahn and Hilliard who proposed to model a multiphase system as a continuous medium and also to define the free energy as a function of concentration and concentration gradient (if the system is isothermal),

$$
f=f_{0}(C)+\frac{\epsilon}{2}(\nabla C)^{2} .
$$

Here $f_{0}$ is the classical part of the free energy, and the second term takes into account the capillary effects. The amplitude of the second term is defined by the so-called capillary parameter $\epsilon$, which is so small that the second term can be neglected everywhere except at the interface, positioned at the places of large concentration gradients. The concentration field $C$ is defined as the mass fraction of one of the liquids in a mixture.

The classical part of the free energy function should define the possible states of the binary mixture. We study the dissolution of an initially heterogeneous mixture, and the classical Landau expression is then convenient to define $f_{0}$,

$$
f_{0}(C)=a\left(C-C_{c}\right)^{2}+b\left(C-C_{c}\right)^{4}
$$


This expression was proposed as an approximation for a near critical system, however, it is used for systems both near and far from the critical point. Thus we treat expression (2) as a model for a binary mixture with homogeneous and heterogeneous states with two phenomenological parameters $a$ and $b$. It can be shown that parameter $a$ is proportional to $\left(T-T_{c}\right)$ : this parameter is negative for the temperatures below the critical point and positive for $T>T_{c}$. The second parameter, $b$, is always positive.

For further discussion, it will be convenient to change the reference point for the concentration field, as $(C-$ $\left.C_{c}\right) \rightarrow C$. The so re-defined concentration will be used in the rest of the paper.

The full Cahn-Hilliard-Navier-Stokes equations that describe the thermo- and hydrodynamic evolution of binary mixtures were first derived by Lowengrub and Truskinovsky [30]. One of the main features of these equations is the quasi-compressibility: even to describe the evolution of two incompressible liquids one needs to use the full continuity equation due to dependence of mixture density on concentration. This feature makes the full set of equations hardly feasible for numerical treatment. It was later shown that the full Cahn-Hilliard-Navier-Stokes equations could however be simplified on the basis of the multiple-scale method by separating fast quasi-acoustic processes and slow diffusive and convective processes [31]. It was shown that the slow evolution of binary mixtures is defined by the Boussinesq approximation, which is used in the current work.

The governing equations include the balances of mass, momentum and species,

$$
\begin{array}{r}
\nabla \cdot \boldsymbol{u}=0, \\
\frac{\partial \boldsymbol{u}}{\partial t}+(\boldsymbol{u} \cdot \nabla) \boldsymbol{u}=-\nabla p+\frac{1}{R e} \nabla^{2} \boldsymbol{u} \\
-C a \nabla^{2} C \nabla C+G r C \boldsymbol{\gamma}, \\
\frac{\partial C}{\partial t}+(\boldsymbol{u} \cdot \nabla) C=\frac{1}{P e} \nabla^{2} \mu, \\
\mu=-G r(\boldsymbol{\gamma} \cdot \boldsymbol{r})+2 A C+4 C^{3}-C a \nabla^{2} C .
\end{array}
$$

Here $p, \boldsymbol{u}$, and $\mu$ are the fields of pressure, velocity and chemical potential; and $\gamma$ is the unit vector defined as $\boldsymbol{g}=-g \boldsymbol{\gamma}$, with $\boldsymbol{g}$ being the gravity acceleration.

The equations written in the Boussinesq approximation are fully incompressible. The diffusive mass transport is defined through the gradient of chemical potential, and includes the effects of barodiffusion. The momentum equation includes the so-called Korteweg force that models the effect of the surface tension on the interface morphology.

The simplest relation between the mixture density and concentration is given for "a simple mixture", when the change in volume upon mixing of two different liquids can be neglected. In addition, we assume that the difference between densities of two pure liquids is small, which is typical for all liquid/liquid mixtures. In such a case, the density is given by

$$
\rho=1-\phi C, \quad \phi \equiv \frac{\rho_{1}-\rho_{2}}{\rho_{1}} .
$$

The equations (3)-(6) are written in non-dimensional form. For time, velocity, pressure, and specific free energy, we choose the following scales:

$$
\tau_{*}=\frac{L_{*}}{V_{*}}, \quad V_{*}=\mu_{*}^{1 / 2}, \quad p_{*}=\rho_{*} \mu_{*}, \quad f_{*}=\mu_{*} .
$$

In these formulae, $L_{*}$ stands for the typical length scale, $\rho_{*}$ is the typical density, e.g. $\rho_{1}$ that is the density of the first component, $\mu_{*}$ is the typical value of the chemical potential, e.g. $\mu_{*}=b$.

The non-dimensional parameters entering the above equations are the Peclet number,

$$
P e=\frac{\rho_{*} L_{*}}{\alpha \mu_{*}^{1 / 2}} ;
$$

the capillary number, ${ }^{1}$

$$
C a=\frac{\epsilon}{\mu_{*} L_{*}^{2}}
$$

the Reynolds number,

$$
R e=\frac{\rho_{*} \mu_{*}^{1 / 2} L_{*}}{\eta_{*}}
$$

and the Grashof number,

$$
G r=\phi \frac{g L_{*}}{\mu_{*}} .
$$

Parameter

$$
A=\frac{a}{\mu_{*}}
$$

defines the equilibrium states of the binary system (if $A$ is negative the system can be homogeneous or heterogeneous in equilibrium, for positive $A$ the binary mixture is always homogeneous in its equilibrium).

In the above expressions, $\alpha$ is the mobility coefficient proportional to the coefficient of diffusion [32]. We need to notice that some new phenomenological parameters are introduced within the phase-field approach, such as the capillary and mobility coefficients. These parameters are used instead of the traditional phenomenological parameters, such as the coefficients of surface tension and diffusion. As a result, the non-dimensional parameters written above have non-standard definitions, and cannot be strictly called by their classical names, Peclet, Reynolds, and Grashof numbers. We however continue to use the classical names, as these parameters appear in front of

\footnotetext{
1 This parameter should not be mixed with another classical parameter, defined as $\eta V / \sigma$, with $\eta, V$, and $\sigma$ being the typical values of viscosity, velocity, and surface tension. This classical parameter is also called the capillary number and is used in the sharp-interface model to define the ratio between the viscous and capillary forces. The introduced parameter is called the capillary parameter to underline that is proportional to the capillary constant, and hence defines the strengths of the capillary effect. In some papers, an alternative name, the Cahn number, is also used [30].
} 


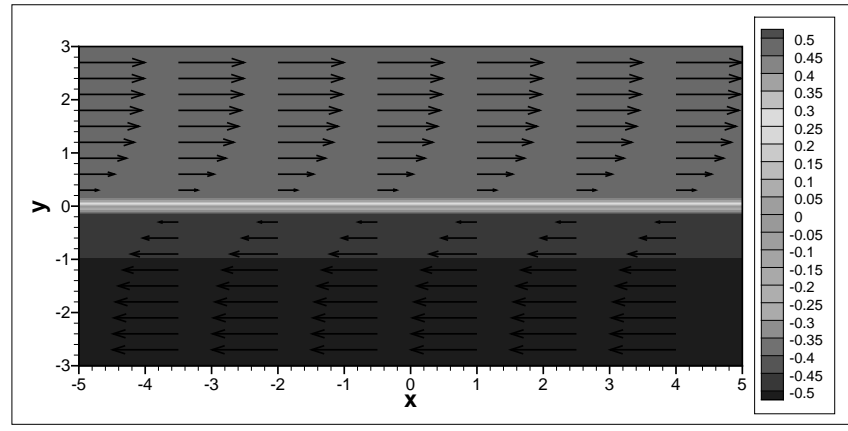

Fig. 1. The basic state is shown for $\delta=0.1, \delta_{u}=1$, and $L=3$.

the corresponding terms of the governing equations (3-6), and hence have similar meanings. For instance, the Peclet number defines the ratio between the convective and diffusive mass transport. The Grashof number defines the intensity of the convective motion, and here, it is defined as the ratio of the buoyancy and inertial terms (which is slightly different from the classical definition that is the ratio of the buoyancy and viscous forces, but such a number will be $G r R e)$. In definition of the Reynolds number, $\eta_{*}$ can be taken as $\frac{1}{2}\left(\eta_{1}+\eta_{2}\right)$ with $\eta_{1}$ and $\eta_{2}$ being the viscosity coefficients of the pure mixture components. ${ }^{2}$

The governing equations need to be supplemented with the boundary conditions. In this work we consider the evolution of a system of two semi-infinite liquid domains. It is assumed that all perturbations should decay at large distances from the interface, which is used to state the boundary conditions for the problem. For numerical integration, the layer's thickness was taken as $2 L$, and gradually increasing values of $L$ were tested in order to find such a value for which the boundary effects do not influence the results.

\subsection{The basic state and linearized equations}

We consider the stability of a horizontal miscible interface that is subjected to an externally driven shear flow. We assume that the interface separates two semi-infinite liquid domains. The basic profiles of concentration and velocity

\footnotetext{
2 The viscosities of mixture components are in general different and hence the mixture viscosity is a function of concentration, which is disregarded in eqs. (3-6). Equations (3-6) were derived in assumption that the state of the system is close to the critical point. When the model (3-6) was derived, viscosity was assumed to depend on concentration. All variables, including concentration and viscosity, were expanded into series of small parameter, and the leading terms of the full CahnHilliard-Navier-Stokes equations formed the model (3-6). The resultant equations are characterised by a constant viscosity coefficient, that can be defined as the average of the viscosities of pure components. The fact that the viscosity can be a function of concentration may result in additional instability [34], which however could not be considered within the framework of eqs. (3-6).
}

are represented by the following functions,

$$
\begin{gathered}
C_{0}=\frac{1}{2} \tanh \left(\frac{y}{\delta}\right), \\
U_{x}=U_{0} \tanh \left(\frac{y}{\delta_{u}}\right) .
\end{gathered}
$$

Thus, $x$-axis is directed along the interface, and $y$-axis is perpendicular to the unperturbed interface. The basic fluid flow has only one non-zero component, and $U_{0}=$ $V_{0} / V_{*}$ is a non-dimensional parameter that defines the flow amplitude $\left(V_{0}\right.$ is the dimensional amplitude of the basic velocity). Two other non-dimensional parameters are the $y$-widths of the concentration and velocity profiles, $\delta$ and $\delta_{u}$. For the most of our further results, the typical length scale is taken to be equal to the width of the basic velocity profile, i.e. $\delta_{u}=1$. Hence, $\delta$ becomes the ratio of the widths of the concentration and velocity profiles. Owing to the fact that a real phase boundary has the thickness of just several molecular layers, the limit of a sharp interface remains always important for the phase-field theory. Thus, we will always assume that $\delta<1$, and will also investigate how the stability results are transformed by reducing the value of $\delta$. The typical basic state is sketched in fig. 1 .

We should also note that for $A<0$ there is another typical interface thickness, $\delta_{0}=\sqrt{-C a / A}$, the thickness of a phase boundary for a binary mixture in the state of thermodynamic equilibrium. In the current work, however, it is assumed that two pure liquids are just brought into contact, and hence the mixture is not in the state of the thermodynamic equilibrium yet. This means that $\delta$ and $C a$ should be treated as two independent parameters. This differs the current work from other studies where the stability of an equilibrium phase boundary is studied [35].

Finally, the surface tension associated with the interface, can be defined as

$$
\sigma=C a \int_{-\infty}^{\infty}\left(\frac{d C_{0}}{d y}\right)^{2} d y=\frac{C a}{3 \delta}
$$

i.e. proportional to the ratio of the capillary number over the interface thickness.

In the current work we investigate the stability of the basic state (14)-(15) in respect to $2 D$ small-amplitude normal disturbances. For this, we split all variable into the following sums of the basic states and perturbations,

$$
\begin{array}{r}
u_{x}(x, y, t) \rightarrow U_{x}(y)+u_{x}(y) \exp (i k x-i \omega t), \\
u_{y}(x, y, t) \rightarrow u_{y}(y) \exp (i k x-i \omega t), \\
C(x, y, t) \rightarrow C_{0}(y)+C(y) \exp (i k x-i \omega t), \\
\mu(x, y, t) \rightarrow \mu_{0}(y)+\mu(y) \exp (i k x-i \omega t), \\
p(x, y, t) \rightarrow p_{0}(y)+p(y) \exp (i k x-i \omega t) .
\end{array}
$$

Note that the same notations are used for the whole variables and for the amplitudes of the disturbances.

Next, we introduce the streamfunction, as $u_{x}=\partial \psi / \partial y$ and $u_{y}=-\partial \psi / \partial x$, and exclude the pressure from the governing equations. The resultant amplitude equations 
then read

$$
\begin{array}{r}
-i\left(\omega-k U_{0}\right)\left(\psi^{\prime \prime}-k^{2} \psi\right)-i k U_{x}^{\prime \prime} \psi= \\
\frac{1}{R e}\left(\psi^{i v}-2 k^{2} \psi^{\prime \prime}+k^{4} \psi\right) \\
-i k C a\left(C_{0}^{\prime \prime \prime} C-C_{0}^{\prime}\left(C^{\prime \prime}-k^{2} C\right)\right)-i k G r C, \\
-i\left(\omega-k U_{x}\right) C-i k C_{0}^{\prime} \psi=\frac{1}{P e}\left(\mu^{\prime \prime}-k^{2} \mu\right), \\
\mu=\left(2 A+12 C_{0}^{2}\right) C-C a\left(C^{\prime \prime}-k^{2} C\right) .
\end{array}
$$

Here primes denote the differentiation in respect to $y$. The eigenvalue problem is solved by using the shooting method with addition of the Gram-Schmidt orthogonalization [3638]. The numerical procedure was based on the integration of the solutions by using the Runge-Kutta method of the fourth order of accuracy with automatically controlled step. The integration was carried out from the left and right ends by using the asymptotic solutions that can be obtained taking in mind that $C_{0}= \pm 1 / 2, U_{x}= \pm U_{0}$ and all derivatives of $C_{0}$ and $U_{x}$ are zero at some distance from the middle point [39-41]. The condition that the eigenfunction needs to be continuous in the middle point $(y=0)$ was used to determine the eigenvalue.

Finally, it is necessary to mention that the basic state, defined by (14) and (15), is in fact time-dependent, as both concentration and velocity profiles slowly smear due to diffusion and viscosity. Nevertheless, the diffusive and viscous smearing would occur considerably slower in comparison with the exponential growth of perturbations, and this allows us to study the evolution of perturbations on the background of the "frozen" basic profiles.

\section{Immiscible liquids}

First, we will examine the stability of an interface separating two immiscible liquids, i.e. for $P e=\infty$. If both $\delta$ and $\delta_{u}$ tend to zero, then the dispersion relation for small perturbations reads [6]

$$
\omega^{2}=\frac{G r}{2} k+\frac{C a}{6 \delta} k^{3}-U_{0}^{2} k^{2} .
$$

Here, $\mathrm{Ca}$ also tends to zero, and the viscous force is neglected.

From formula (25) one concludes that if $G r<0$ (the heavier liquid is on top of the lighter one) the interface is unstable owing to the Rayleigh-Taylor instability (the first term), and, in the presence of the flow, the interface can be additionally unstable owing to the Kelvin-Helmholtz instability (the third term). The capillary effect (the second term) suppresses the development of the short-wave modes.

In the opposite gravitationally stable case, $G r>0$, the perturbations result in development of the gravitycapillary waves for higher Grashof number (weak flows). The interface can however disintegrate due to the KelvinHelmholtz instability if a sufficiently strong shear flow is imposed.
These are the classical conclusions for infinitely thin interfaces. A smeared interface is, in addition, prone to the Holmboe instability (for $G r>0$ ) [13]. For the smeared interface the amplitude equations (22)-(24), if simplified by neglecting the capillary and viscous terms, are reduced to the classical Taylor-Goldstein equation $[17,24]$,

$$
\psi^{\prime \prime}-\left(k^{2}-\frac{k U_{x}^{\prime \prime}}{\left(\omega-k U_{x}\right)}+\frac{k^{2} J(y)}{\left(\omega-k U_{x}\right)^{2}}\right) \psi=0,
$$

where $J(y)=C_{0}^{\prime} G r$ is the squared Brunt-Vaisala frequency.

The stability analysis of this equation was performed for various density and velocity profiles. It was proven that the layer is stable if the global Richardson number,

$$
R i(y)=\frac{J(y)}{\left[U_{x}^{\prime}\right]^{2}}=\frac{C_{0}^{\prime} G r}{\left[U_{x}^{\prime}\right]^{2}}=\frac{G r \delta_{u}^{2}}{2 \delta} \frac{\cosh ^{4}\left(y / \delta_{u}\right)}{\cosh ^{2}(y / \delta)}
$$

is everywhere greater than $1 / 4$. If somewhere $\operatorname{Ri}(y) \leq 1 / 4$ then instability is likely to occur but this is not guaranteed, and the stability analysis is required for a definite answer [17]. For the basic profiles (14) and (15) formula (27) defines that the stability is determined by the Grashof number and, in addition, by the ratio $\delta / \delta_{u}$. We generally assume that $\delta_{u}>\delta \rightarrow 0$. In this case, the instability is to be expected for $\delta<\delta_{u} / 2\left(\delta<1 / 2\right.$ if $\left.\delta_{u}=1\right)$, irrespectively of the value of the Grashof number.

\section{1 $G r<0$ : the lighter liquid is superposed by the heavier one}

If $G r<0$, a horizontal phase boundary is always unstable due to the Rayleigh-Taylor instability. If, in addition, the neighbouring liquids participate in a relative motion, the Rayleigh-Taylor instability competes with the KelvinHelmholtz instability. This is illustrated in fig. 2a, where the increase in the intensity of the imposed flow leads to a stronger growth of the modes with the longer wavelengths, and to a weaker growth of the modes with the shorter wavelengths. Thus, the instabilities do not sum up, as some modes develop slower, but this result agrees well with the simple analytic formula (25) for sharp interfaces and also with the earlier studies [8-10], in which the stability of smeared interfaces was previously examined. Figure 2a also depicts the data for the inviscid and viscous liquids, showing the dissipative role of the viscous force.

Figures 2b,c show the growth rates for perturbations developing at the interfaces of different thicknesses for the cases of $C a=0(2 \mathrm{~b})$ and $C a=0.001(2 \mathrm{c})$. The results are similar to what we earlier observed for the case of the pure Rayleigh-Taylor instability (see ref. [1]). Namely, for $C a=0$, we notice that the curves converge to the limit of sharp interface behaviour. If however the capillary effects are non-neglected, a simple reduction in the interface thickness leads to the growth of the surface tension (which is proportional to $C a / \delta$, see (16) and (25)) and to the strong damping of the short wavelength modes. For 


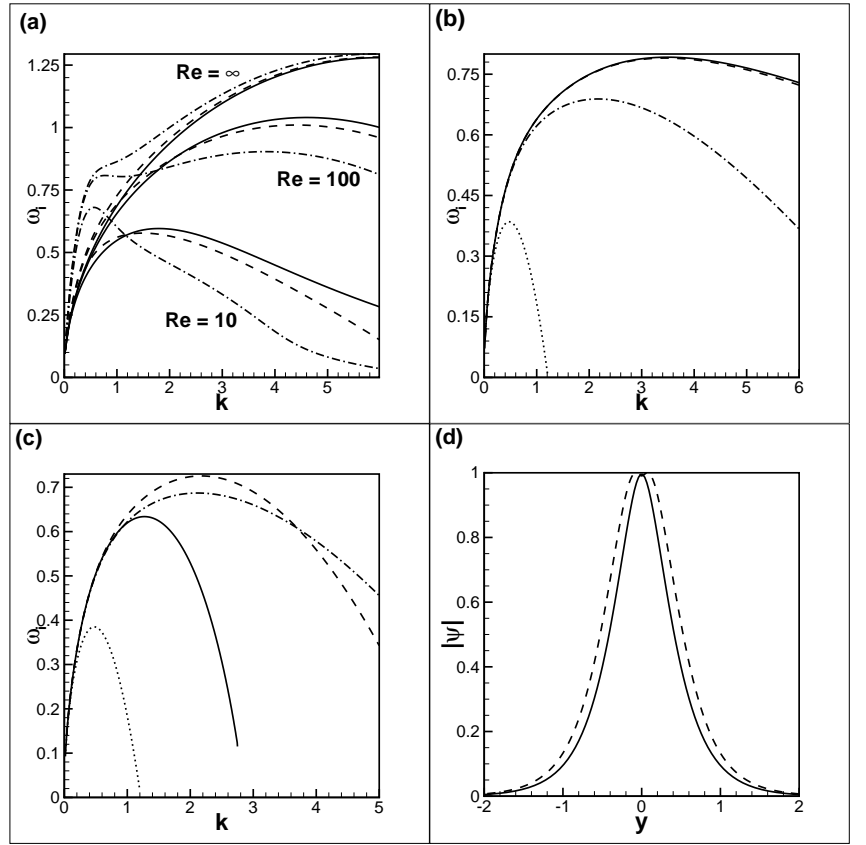

Fig. 2. (a-c) The growth rates vs. the wavenumber are shown for an immiscible interface that separates the heavier liquid lying over the lighter liquid. The curves are plotted for $G r=$ $-1, \delta_{u}=1$ and (a) $C a=0.001, \delta=0.05, U_{0}=0$ (solid lines), $U_{0}=1$ (dashed lines), and $U_{0}=3$ (dash-dot lines) for three different Reynolds numbers, $R e=\infty, R e=100$, and $R e=10 ;$ (b) $C a=0, R e=25, U_{0}=1$, and $\delta=0.001$ (solid line), $\delta=0.01$ (dashed line), $\delta=0.1$ (dash-dot line), and $\delta=1$ (dotted line); (c) $C a=0.001, R e=25, U_{0}=1$, and different $\delta$ (lines as in (b)). (d) The eigenfunctions, namely the moduli of the streamfunctions, are plotted for $k=3, G r=-1$, $R e=25, U_{0}=1, \delta=0.01, C a=0$ (solid line) and $C a=0.01$ (dashed line). The eigenvalues are $\omega=(0,0.750)$ for $C a=0$, and $\omega=(0,0.531)$ for $C a=0.01$.

the pure Rayleigh-Taylor instability it was shown, that the sharp interface behaviour can be revealed by simultaneous decrease of the interface thickness and capillary number. This dependency however was not reproduced for the interface with the imposed shearing flow.

Finally, fig. 2d shows the shapes of the eigefunctions, i.e. the $y$-profiles of the streamfunctions for two cases of $C a=0$ and $C a=0.001$. In terms of the eigenfunctions, the difference between the cases of zero and non-zero capillary effects is not very strong. The eigenfunctions plotted for the interfaces of different thicknesses were also found to be almost coincident.

\section{2 $G r>0$ : the lighter liquid superposes the heavier one}

The configuration with the heavier liquid lying underneath the lighter liquid would obviously be stable without externally imposed flow, and the flow could make this configuration unstable towards the Kelvin-Helmhotz and Holmboe instabilities. The stability diagrams are depicted in fig. 3. The Kelvin-Helmhotz instability is limited to the
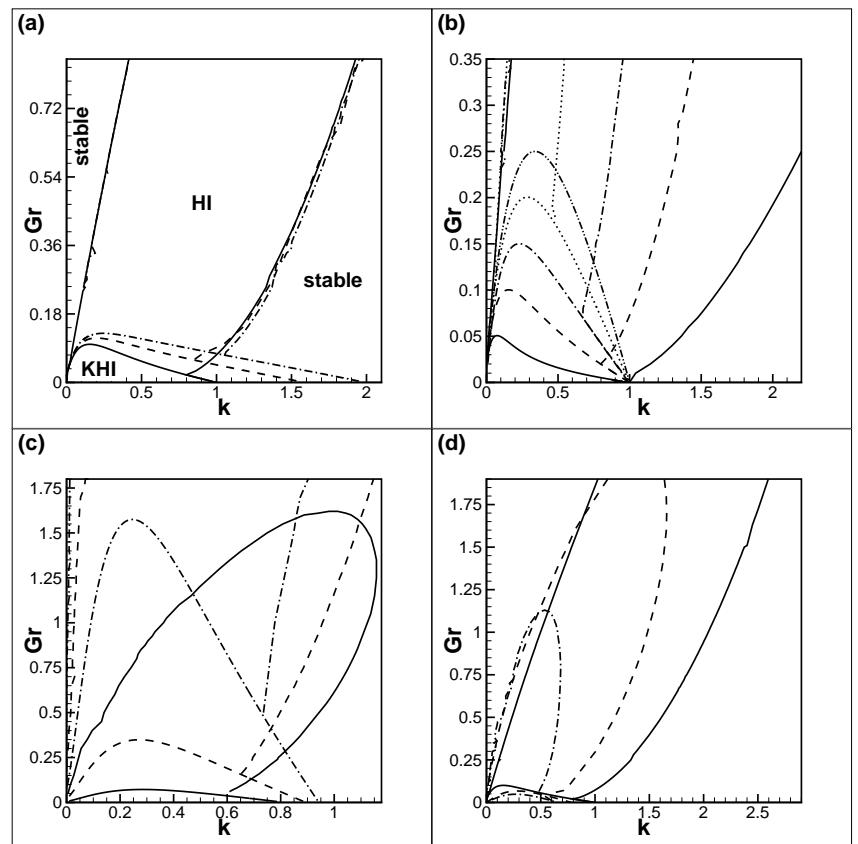

Fig. 3. The neutral curves defining the zones of the KelvinHelmoltz (maked by "KHI") and Holmboe ("HI") instabilities. The results are obtained for an immiscible $(P e=\infty)$ horizontal interface separating two liquids with the heavier liquid at the bottom. The parameters are (a) $\delta=0.2, U_{0}=1, R e=\infty$, and $C a=0$ (solid line), $C a=0.0005$ (dashed line), and $C a=0.001$ (dash-dot line); (b) $R e=\infty, U_{0}=1$, and $\delta=0.1$ (solid line), $\delta=0.2$ (dashed line), $\delta=0.3$ (dash-dot line), $\delta=0.4$ (dotted line), and $\delta=0.5$ (dash-dot-dot line); (c) $\delta=0.2, R e=20$, and $U_{0}=1$ (solid line), $U_{0}=2$ (dashed line), and $U_{0}=4$ (dash-dot line); (d) $\delta=0.2, U_{0}=1$, and $R e=\infty$ (solid line), $R e=50$ (dashed line), and $R e=10$ (dash-dot line). $\delta_{u}=1$ for all plots.

lower Grashof numbers (smaller density contrasts), while the evolution at the higher Grashof numbers is dominated by the Holmboe instability. The zone of the Holmboe instability is usually large (in comparison to the zone of the Kelvin-Helmholtz instability), and even remains unclosed from the top for immiscible interfaces between inviscid liquids, when the growth of unstable modes is not stabilised even by the strong gravity force. Similar stability diagrams were earlier reported by other researchers $[42,18]$.

Figure 3a shows the stability diagrams for the capillary forces of different strengths. Surprisingly, the increase in the surface tension does not make the layer more stable, and, on the contrary, the capillary forces extend the range of the Kelvin-Helmholtz instability to the modes with shorter wavelengths, and slightly widens the zone of the Holmboe instability.

Figure $3 \mathrm{~b}$ depicts the neutral curves for the interfaces of different thicknesses. Thicker interfaces are more sensitive to the Kelvin-Helmholtz instability. The range of Holmboe instability is however decreased for such interfaces, and, for the case of $C a=0$ and $U_{0}=1$, the Holmboe modes cease to exist if $\delta>0.417$. In the opposite limit of thinner thicknesses, the Kelvin-Helmholtz instability be- 

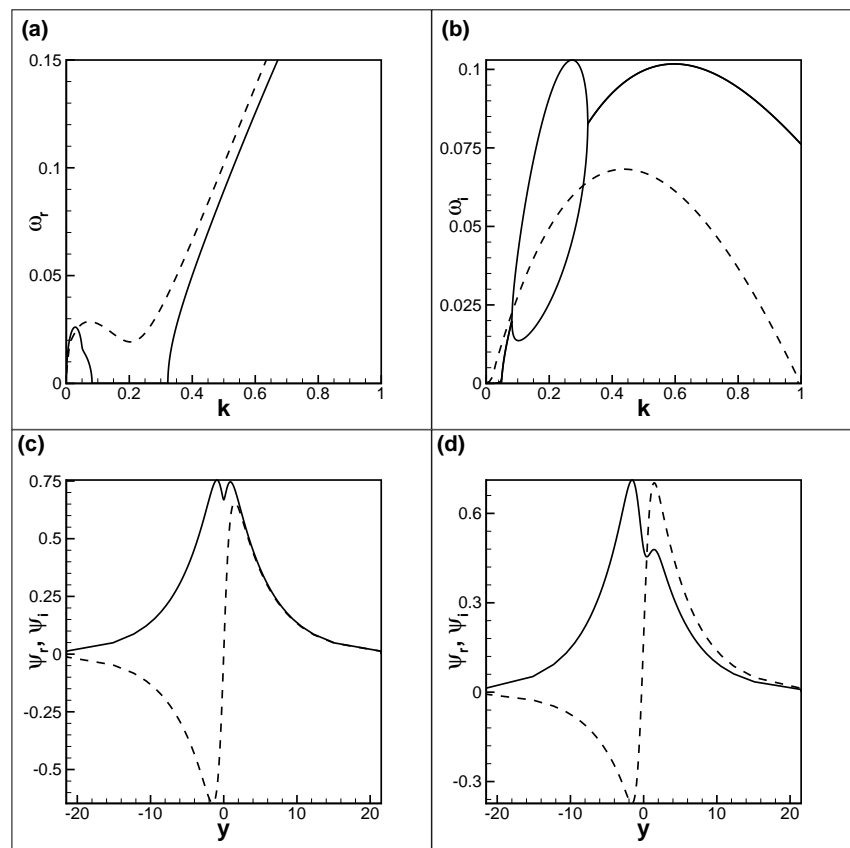

Fig. 4. $(\mathbf{a}, \mathbf{b})$ The eigenspectra for the perturbations developing at an immiscible $(P e=\infty)$ horizontal interface with the heavier liquid underneath the lighter one. The curves are shown for $G r=0.1, C a=0, \delta=0.2, R e=\infty$ (solid lines) and $R e=50$ (dashed lines). (a) shows $\omega_{r}$ and (b) shows $\omega_{i} .(\mathbf{c}, \mathbf{d})$ The eigenfunctions, namely, the real (solid lines) and imaginary (dashed lines) parts of the streamfunction, are plotted for $k=0.2, G r=0.1, C a=0, \delta=0.2$, and (c) $R e=\infty$, the eigenvalue is $\omega=(0,0.094)$; and (d) $R e=50, \omega=(0.022,0.05)$. $\delta_{u}=1$ for all plots.

comes strongly suppressed and the instability is primarily defined by the Holmboe modes.

Figure $3 \mathrm{c}$ shows how the increase in the amplitude of the imposed flow affects the shapes of the neutral curves, and, as expected, the zones of the both Kelvin-Helmholtz and Holmboe instabilities grow. The curves are plotted for the finite value of the Reynolds number, and, by making comparison against fig. 3a, one notices that viscosity makes the zones of the Holmboe instability closed up, so the instability becomes suppressed by stronger gravity. Figure $3 \mathrm{~d}$ defines the stability diagrams for the different strengths of the viscous forces, additionally illustrating that viscosity reduces the range of unstable modes.

The typical eigenspectra are depicted in fig. 4a,b. The results are shown for the parameters of fig. $3 \mathrm{~b}$, for $G r=$ 0.1 , and for the inviscid and viscous cases. In these plots, one sees that there is a short range of stable modes closer to $k=0$, then there is a range of unstable modes (for which $\omega_{i}$ is positive), and again the instability is reinstated at larger wavenumbers. The Kelvin-Helmholtz modes have zero wavespeeds $\left(\omega_{r}=0\right)$, and the Holmboe modes are characterised by non-zero speeds (the positive branch of $\omega_{r}$ is only shown, and there is another symmetric branch with negative $\omega_{r}$ defining the wave travelling in the opposite direction). The development of the Kelvin-Helmholtz modes can be suppressed by viscosity (like shown in fig. 4b), nevertheless, the modes remain unstable in respect to the Holmboe instability.

Figures 4c,d depict the typical shapes of eigenfunctions. Figure 4c depicts the Holmboe mode and fig. 4d depicts the Kelvin-Helmholtz mode, the shapes of which are however not substantially different. The eigenfunctions are much wider in comparison to the similar functions plotted for the gravitationally unstable configuration (fig. 2d). In addition, there appears some non-symmetry about the middle point.

\section{Miscible liquids}

\section{1 $G r<0$ : the lighter liquid is superposed by the heavier one}

For miscible liquids, the configuration with the heavier liquid on top of the lighter liquid remains unconditionally unstable. Figures 5, 6, and 7 show the growth rates as functions of the wavenumber for various values of the nondimensional parameters. Figure 5 depicts the results obtained for $C a=0$, and figs. 6 and 7 illustrate the changes in the eigenspectra enforced by the capillary terms. In general, we may conclude that the effect of capillarity is mostly reduced to damping of the shortwave modes.

Figure 5a shows that the diffusion slows down the growth of perturbations. The viscosity also plays the dissipating role, which is illustrated in figs. 5c and $7 \mathrm{a}$.

Figures $5 \mathrm{~b}$ and $6 \mathrm{a}, \mathrm{b}$ show the growth rates for the perturbations developing at interfaces of different thicknesses. In the case of negligible capillary effects (fig. 5b) the results could be obtained for the very small values of interface thickness, and convergence of the curves defining the sharp interface limit could be observed. If however the capillarity is important (figs. 6a,b), then the curves for much thicker interfaces could only be obtained, and convergence to the sharp interface behaviour was not revealed. In addition, we plotted the curves for the different values of $C a$ and $\delta$, so that their values are simultaneously reduced (figs. 6c,d). In these plots, the limiting behaviour of the sharp interface was not revealed either. As already mentioned above, the ratio $\mathrm{Ca} / \delta$ defines the surface tension coefficient, and the simultaneous changes in these two parameters allowed us to obtain the behaviour of a sharp interface in the case of the pure Rayleigh-Taylor instability $\left(U_{0}=0\right)$. The flow imposed along the interface obviously makes the dependence of the eigenspectrum on $\mathrm{Ca}$ and $\delta$ more complex.

Figures 5d and 7c show how the flow amplitude affects the eigenspectrum. Similar to the immiscible case, the growth of the modes with long wavelengths is intensified, and the growth of the modes with the shorter wavelengths is reduced.

Figures 5e and $7 \mathrm{~d}$ show that the value of the parameter $A$ does not introduce any qualitative changes to the eigenvalue spectrum. This parameter defines the effective diffusion coefficient, $D_{0}=2 A+12 C_{0}^{2}$, and hence, the larger values of $A$ correspond to stronger diffusion, and, consequently, to stronger damping of the growth. Finally, fig. 


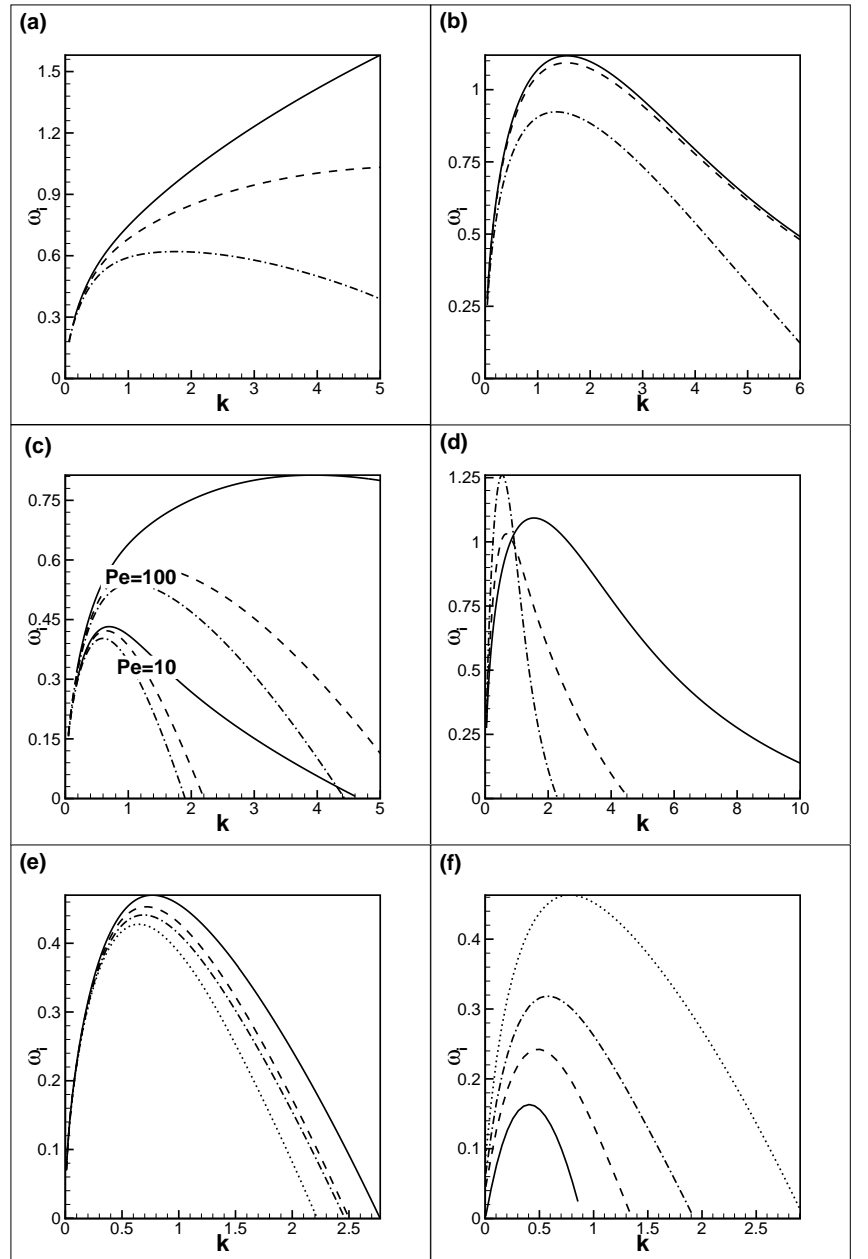

Fig. 5. The growth rates vs. the wavenumber are shown for a miscible interface separating two liquids with the lighter liquid at the bottom. The curves are plotted for $C a=0, \delta_{u}=1$ and (a) $G r=-1, \delta=0.1, R e=\infty, A=-0.5, U_{0}=1$, and for $P e=\infty$ (solid line), $P e=250$ (dashed line), and $P e=50$ (dash-dot line); (b) $G r=-1, P e=20, R e=\infty$, $A=0.5, U_{0}=1$, and for $\delta=0.001$ (solid line), $\delta=0.01$ (dashed line), and $\delta=0.1$ (dash-dot line); (c) Gr $=-0.5$, $U_{0}=1, \delta=0.1, P e=10$ and $P e=100$ and $R e=\infty$ (solid lines), $R e=50$ (dashed lines), and $R e=25$ (dash-dot lines), (d) $G r=-1, \delta=0.01, P e=20, R e=\infty$, and $U_{0}=1$ (solid line), $U_{0}=3$ (dashed line), and $U_{0}=5$ (dash-dot line), (e) $G r=-1, \delta=0.01, P e=20, R e=50$, and $A=-0.5$ (solid line), $A=-0.25$ (dashed line), $A=0.25$ (dash-dot line), and $A=0.5$ (dotted line); (f) $U_{0}=1, P e=20, R e=50, A=0.5$, $\delta=0.1$, and $G r=0$ (solid line), $G r=-0.25$ (dashed line), $G r=-0.5$ (dash-dot line), and $G r=-1$ (dotted line).

5f shows the role of the gravity force on the growth rates. In the presence of the flow, the instability is obviously observed even for $G r=0$, when the phase boundary is subject to the pure Kelvin-Helmholtz instability. The range of the unstable modes and the maximum growth rates are increased if the Grashof number is increased and hence the Rayleigh-Taylor instability is intensified.
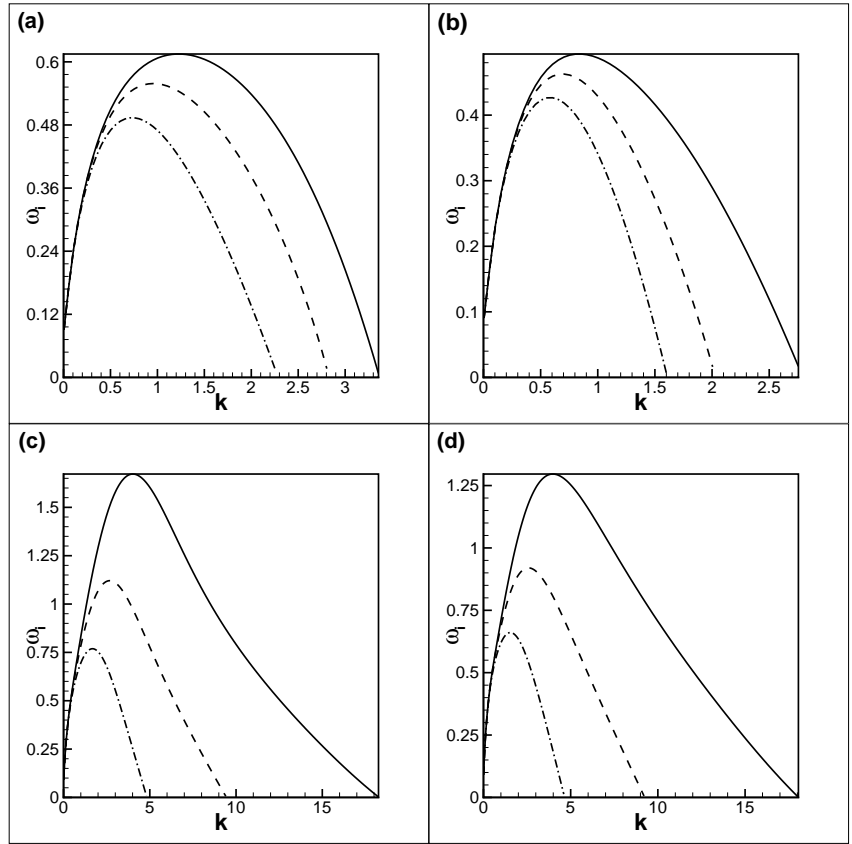

Fig. 6. The growth rates vs. the wavenumber are shown for a miscible interface between two liquids with the lighter liquid at the bottom. The curves are plotted for $\delta_{u}=1, G r=-1$, $U_{0}=1, P e=20$, (a) $C a=0.05, R e=\infty, A=-0.5, \delta=0.3$ (solid line), $\delta=0.5$ (dashed line), and $\delta=0.8$ (dash-dot line); (b) $R e=\infty, C a=0.05, A=0.5$ and for different $\delta$ (lines marked as in (a)); (c) $R e=20, A=-0.5$, and $C a=\delta=0.05$ (solid line), $C a=\delta=0.1$ (dashed line), $C a=\delta=0.2$ (dashdot line); (d) $R e=20, A=-0.5$, and values of $C a$ and $\delta$, and lines as in (c).

Figure 8 depicts the typical shapes of the eigenfunctions, namely, the moduli of concentration and streamfunction. The eigenfunctions are shown for two values of parameter $A$ and for two different thicknesses $\delta$. The concentration profiles are noticeably narrower and become even more narrower if the interface thickness is reduced. The streamfunction profiles remain virtually unaffected by the changes in $A$ and $\delta$, but we saw that the streamfunction profiles become narrower for lower values of the Reynolds numbers (stronger viscosity).

\section{2 $G r>0:$ the lighter liquid above the heavier one}

Figures 9, 11, and 12 depict the neutral curves that define the zones of the Kelvin-Helmholtz and Holmboe instabilities obtained for interfaces separating two miscible liquids. Figure 9 shows the results for $C a=0$. Figures $9 \mathrm{a}, \mathrm{b}$ are obtained for rather thin interfaces with and without interfacial diffusion. In the immiscible case, the zone of the Kelvin-Helmholtz instability is small, but the layer is almost unconditionally unstable to the Holmboe modes, which agrees with the observations made from fig. $3 \mathrm{~b}$ plotted for the immiscible interfaces of different thicknesses. An introduction of interfacial diffusion changes the stability boundaries: the zone of the Kelvin-Helmholtz instability gets enlarged, while the zone of the Holmboe instability 

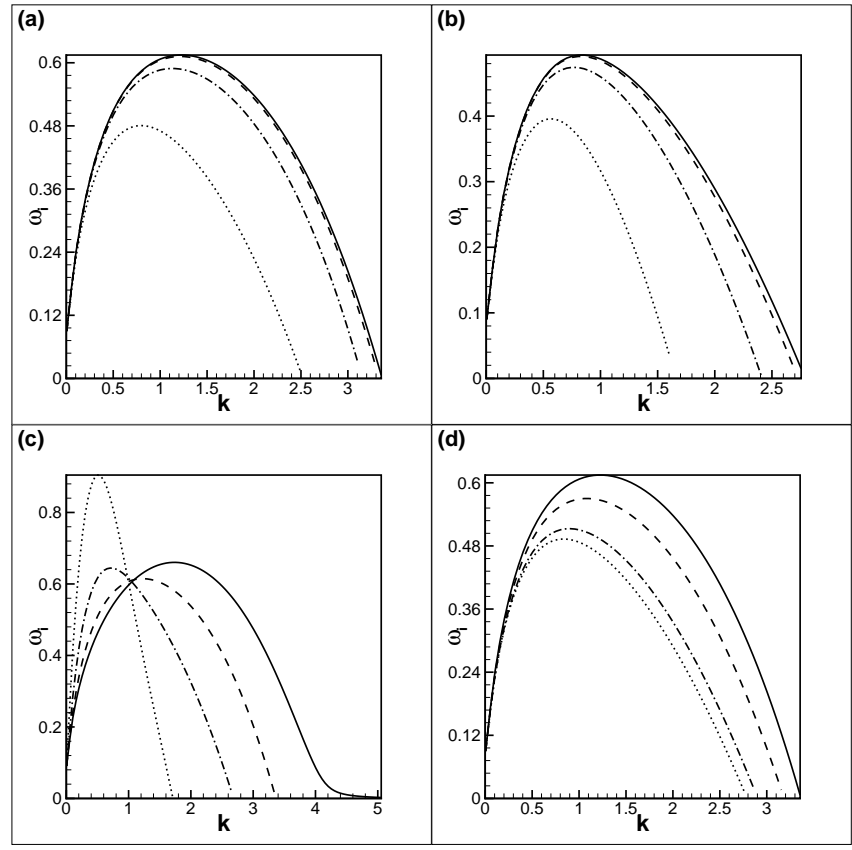

Fig. 7. The growth rates vs. the wavenumber are shown for a miscible interface between two liquids with the lighter liquid at the bottom. The curves are plotted for $G r=-1, P e=20$, $C a=0.05, \delta=0.3, \delta_{u}=1$, (a) $A=-0.5, U_{0}=1, R e=\infty$ (solid line), $R e=1000$ (dashed line), $R e=100$ (dash-dot line), and $R e=10$ (dotted line); (b) $A=0.5, U_{0}=1$, and different $R e$ (lines as in (a)); (c) $R e=\infty, A=-0.5, U_{0}=0$ (solid line), $U_{0}=1$ (dashed line), $U_{0}=2$ (dash-dot line), $U_{0}=4$ (dotted line); and (d) $U_{0}=1, R e=\infty, A=-0.5$ (solid line), $A=-0.25$ (dashed line), $A=0.25$ (dash-dot line), and $A=0.5$ (dotted line).

becomes narrower. Thus, in the case of strong interfacial diffusion (figs. 9c,d), the thinner interfaces are more unstable. In these figures, the zones of the Holmboe instability are unclosed, with the left boundaries virtually coincident with the axis $k=0$.

Figure 10 depicts the typical eigenspectra. Both the real and imaginary parts of the eigenvalues are plotted. The imaginary part defines the growth or decay rates of perturbations, so that its positive values signify the instability. One may notice a thin region near $k=0$, where the perturbations decay, then, there is a region of the KelvinHelmholtz $\left(\omega_{r}=0\right)$ or Holmboe $\left(\omega_{r} \neq 0\right)$ instabilities, and the shortwave modes decay again. The results are shown for the viscous and inviscid cases, and one sees that the instability region is reduced with the added viscous effect. In addition, the Kelvin-Helmholtz instability can be observed for higher values of $R e$ numbers, but it ceases to exist at such a level of the Grashof number if the viscosity is stronger.

Figures 11 and 12 show the neutral curves for miscible interfaces with the full account of the capillary terms. As expected, the interface is stable for higher Grashof numbers. At lower values of the Grashof number the interface is subject to either the Kelvin-Helmhotz or Holmboe instabilities. The effect of interfacial diffusion on the interface
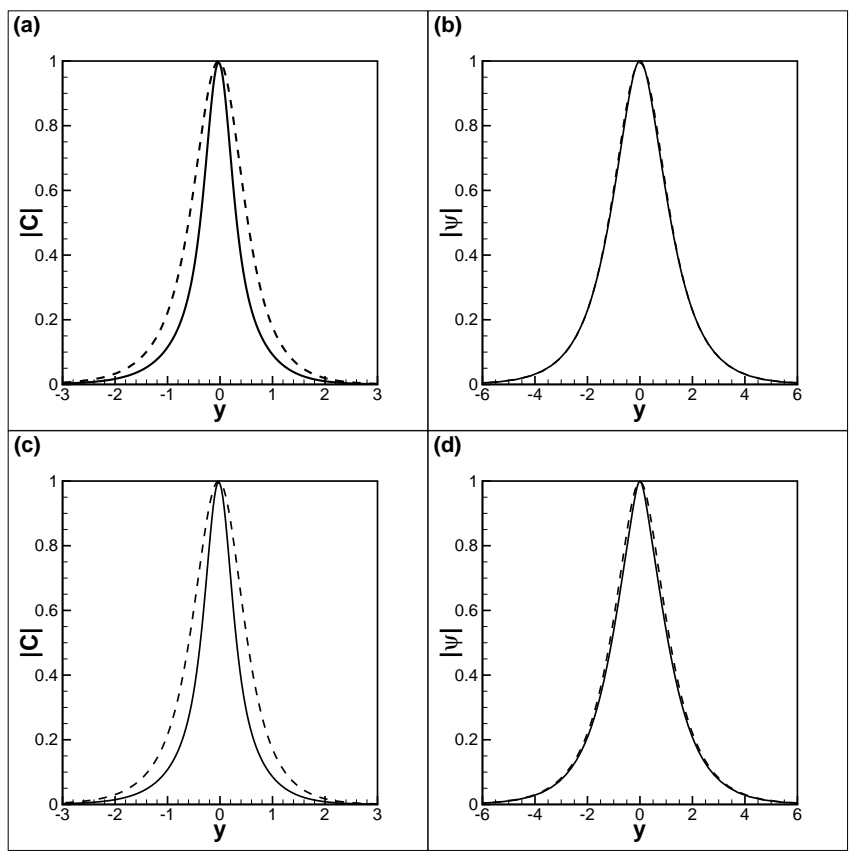

Fig. 8. The eigenfunctions, namely the moduli of the concentration $(\mathbf{a}, \mathbf{c})$ and streamfunction $(\mathbf{b}, \mathbf{d})$ are shown for $k=1$, $G r=-1, P e=20, C a=0.05, \delta_{u}=1, U_{0}=1, R e=\infty$, $\delta=0.3$ (solid lines) and $\delta=0.8$ (dashed lines). The results are plotted for $A=-0.5(\mathbf{a}, \mathbf{b})$ and $A=0.5(\mathbf{c}, \mathbf{d})$. The eigenvalues are $(\mathbf{a}, \mathbf{b}) \omega_{i}=0.607(\delta=0.3), \omega_{i}=0.469(\delta=0.8)$ and $(\mathbf{c}, \mathbf{d})$ $\omega_{i}=0.487(\delta=0.3), \omega_{i}=0.341(\delta=0.8)$.

stability is illustrated by figs. 11a,b. The influence of diffusivity is different for the negative and positive values of parameter $A$. If $A<0$, then diffusion through the interface suppresses the development of the Kelvin-Helmoltz instability, but the zone of the Holmboe instability gets even larger. The Holmboe instability does not exist for the cases of weak diffusivity (large $P e$ numbers). If $A>0$, the range of $G r$ for which the instability can be observed is much larger. The presence of interfacial diffusion does not significantly change the zone of the Kelvin-Helmholtz instability until diffusion is too strong $(P e=10)$, see fig. 11b. The increase in diffusivity makes the region of the Holmboe instability smaller, and the Holmboe instability is not observed for low Peclet numbers (e.g. Pe =10).

Figures 11c,d show the neutral curves for the interfaces of various thicknesses. Similar to fig. 9c,d thinner interfaces are more prone to the Kelvin-Helmholtz instability. In ref. [1], it was shown that the configuration with the heavier liquid below the lighter one is thermodynamically unstable with the exponential growth of perturbations for the interfaces thicker than the equilibrium value $\delta_{0} \equiv \sqrt{-C a / A}$. This result is confirmed in the current work and is illustrated in fig. 11c, where the neutral curve for $\delta=2 \delta_{0}$ splits the plot into two parts: the longwave modes are thermodynamically unstable (almost independent on the value of the Grashof number), and the shortwave modes are stable. We did not observe the convergence in the results to the sharp interface behaviour for the limit of zero interface thicknesses. 


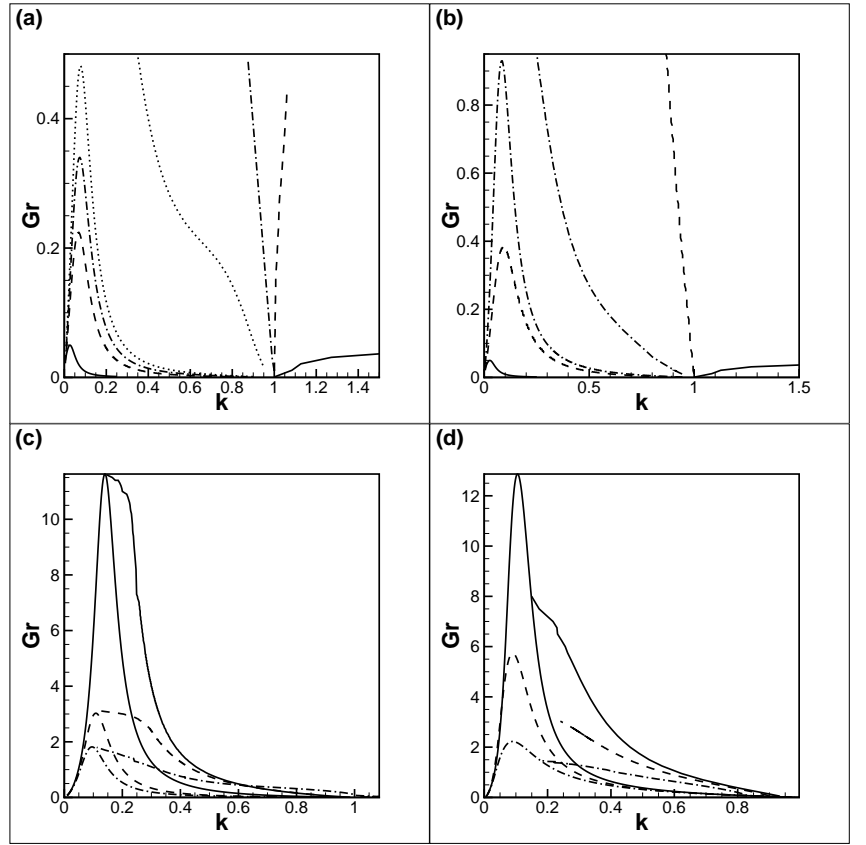

Fig. 9. The neutral curves are plotted for a miscible interface between two inviscid liquids $(R e=\infty)$ with the heavier liquid underneath the lighter one. The capillary effects are disregarded $(C a=0)$. Other parameters are $\delta_{u}=1, U_{0}=1,(\mathbf{a})$ $A=-0.5, \delta=0.01, P e=\infty$ (solid line), $P e=1000$ (dashed line), $P e=400$ (dash-dot line), and $P e=200$ (dotted line); (b) $A=0.5, \delta=0.01, P e=\infty$ (solid line), $P e=2500$ (dashed line), $P e=100$ (dash-dot line); (c) $P e=20, A=-0.5$, $\delta=0.001$ (solid line), $\delta=0.005$ (dashed line), $\delta=0.01$ (dashdot line); (d) $P e=10, A=0.5, \delta=0.05$ (solid line), $\delta=0.1$ (dashed line), and $\delta=0.2$ (dash-dot line).

Figures 11e,f show that viscosity introduces additional damping thus reducing the range of unstable modes.

Figure 12a depicts the neutral curves for different $A$. The more positive values of $A$ (stronger diffusion) correspond to larger zone of the Kelvin-Helmholtz instability, which agrees with figs. 11a,b. Correspondingly, at negative $A$, the zones of instability are significantly reduced. Figure $12 \mathrm{~b}$ illustrates that the capillary terms enlarge the zones of instability, similar to the immiscible case (fig. 3a). Figures $12 \mathrm{c}, \mathrm{d}$ show the neutral curves for the imposed flows of different amplitudes. Obviously, the increase in the flow intensity expands the zones of the Kelvin-Helmholtz and Holmboe instabilities.

Finally, fig. 13 depicts the eigenfunctions plotted for the parameters of fig. $11 \mathrm{~d}$, for $k=0.2$, for two different levels of Grashof numbers that correspond to the KelvinHelmoltz and Holmboe disturbances. The streamfunction profiles look very similar to the curves plotted in fig. $4 \mathrm{c}, \mathrm{d}$ for the immiscible results. In the miscible case, the concentration profiles are added. The concentration profiles are much narrower, with the width similar to the case of $G r<0$ (fig. 8a,c)
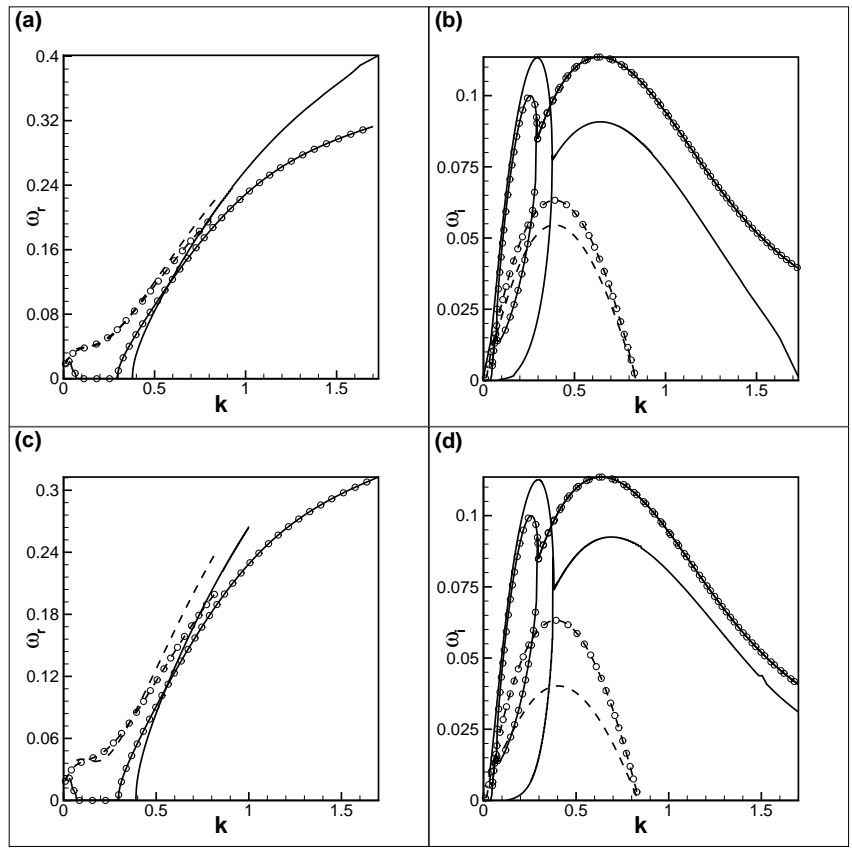

Fig. 10. The eigenspectra are shown for the perturbations developing at a miscible interface that separates two liquids with the heavier liquid underneath. The two sets of curves are plotted for $R e=20$ (dashed lines) and $R e=\infty$ (solid lines). Other parameters are $G r=0.1, \delta=0.01, \delta_{u}=1, U_{0}=1$, and $(\mathbf{a}, \mathbf{b}) A=-0.5, P e=1000$ and $P e=\infty$ (lines marked by $\circ$ symbols); (c,d) $A=0.5, P e=2500$ and $P e=\infty$ (lines marked by $\circ$ symbols $)$.

\section{Conclusions}

The stability of a phase boundary separating two miscible liquids is studied. The liquids are two components of a non-equilibrium binary mixture, with the phase boundary modelled by a sharp transition in concentration. The shear flow is imposed along the interface, represented by a similar transition in velocity, but with a different thickness. Both basic concentration and velocity profiles do not represent the equilibrium solutions, and thus both profiles experience slow smearing due to diffusivity and viscosity. From the governing equations (3-6), it could be understood that the smearing rates are proportional to $(t / P e)^{1 / 2}$ and $(t / R e)^{1 / 2}$ (the convective and diffusive time scales are assumed to be comparable within this work). The aim was to understand whether a faster and more complex mixing can overrun the simple interface smearing.

We used the phase-field approach to investigate the linear stability of a horizontal interface. The base profiles were perturbed with normal disturbances. One understands that both the diffusive spreading of the base state and the growth of the perturbations are defined by the similar time scales. Nevertheless, the spreading occurs considerably slower due to different time-dependencies: the spreading occurs as $t^{1 / 2}$, while the perturbations grow exponentially. This allowed us to consider the evolution of perturbations on the background of the "frozen base 

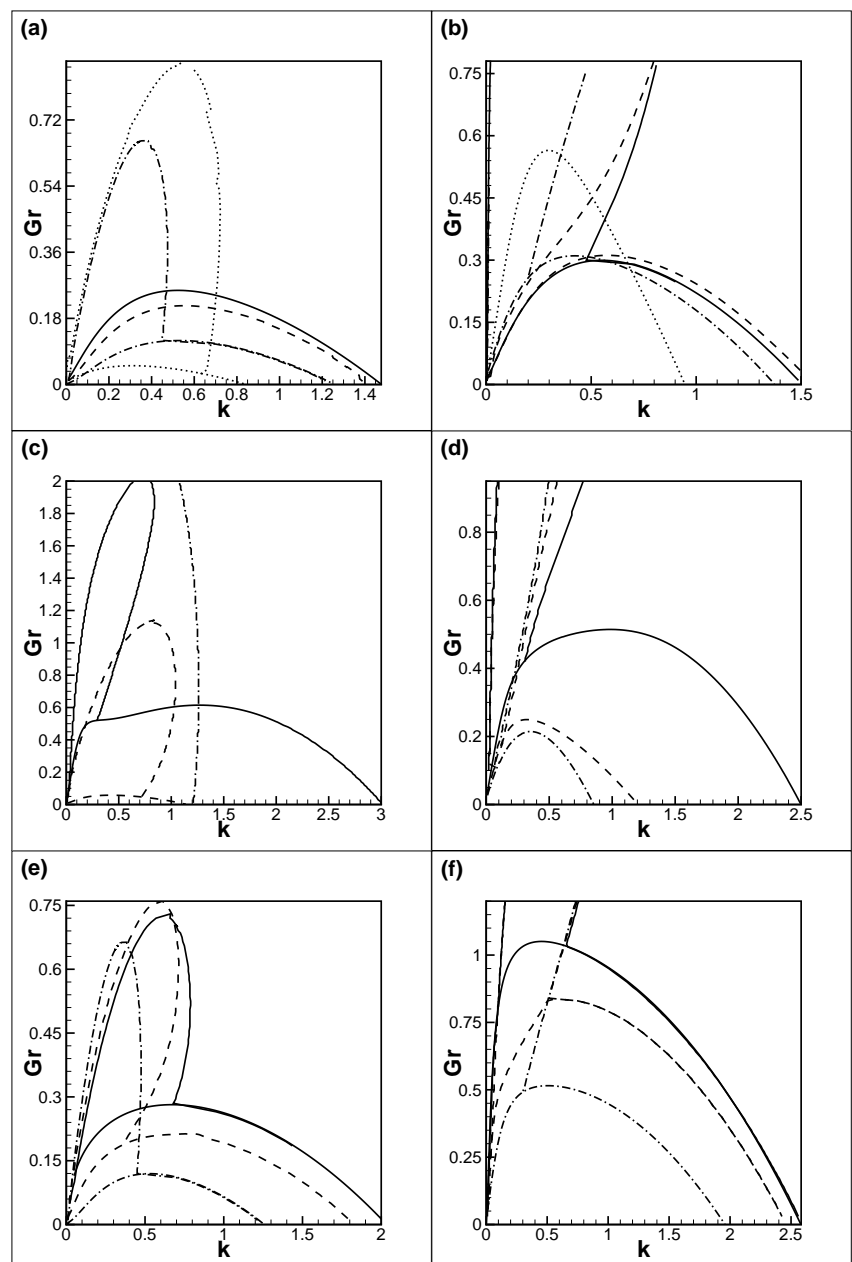

(d)

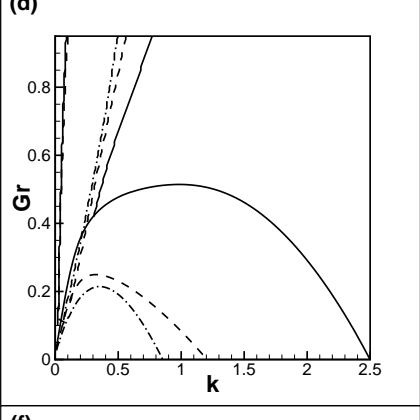

(f)

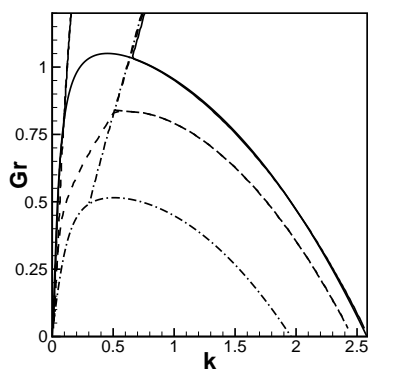

Fig. 11. The neutral curves are plotted for a miscible interface between two liquids with the heavier one underneath. The curves are obtained for $U_{0}=1, \delta_{u}=1$ and (a) $R e=25$, $\delta=0.3, A=-0.5, C a=0.05, P e=\infty$ (solid line), $P e=1000$ (dashed lines), $P e=100$ (dash-dot lines), and $P e=10$ (dotted lines); (b) $A=0.5$, other parameters and lines as in (a); (c) $P e=20, R e=100, A=-0.5, C a=0.05, \delta=\delta_{0} / 2 \approx 0.158$ (solid lines), $\delta=\delta_{0} \approx 0.316$ (dashed lines), $\delta=2 \delta_{0} \approx 0.632$ (dash-dot line); (d) $P e=100, R e=25, A=0.5, C a=0.05$, $\delta=0.1$ (solid lines), $\delta=0.3$ (dashed lines), and $\delta=0.6$ (dashdot lines); (e) $P e=100, \delta=0.3, A=-0.5, C a=0.05$, $R e=\infty$ (solid lines), $R e=250$ (dashed lines), and $R e=25$ (dash-dot lines); (f) $A=0.5, C a=0.08$, other parameters and lines as in (e).

state", which is the general approach commonly used for solving the eigenvalue problems with slowly variable coefficients. The obtained results were validated against the data available for immiscible interfaces that are subject to either the Rayleigh-Taylor, or Kelvin-Helmholtz, or Holmboe instabilities.

The results are obviously different for the gravitationally stable and unstable configurations. In the case of the heavier liquid lying above the lighter liquid, the interface is unconditionally unstable. The addition of the flow does not make the interface more unstable, in sense, that
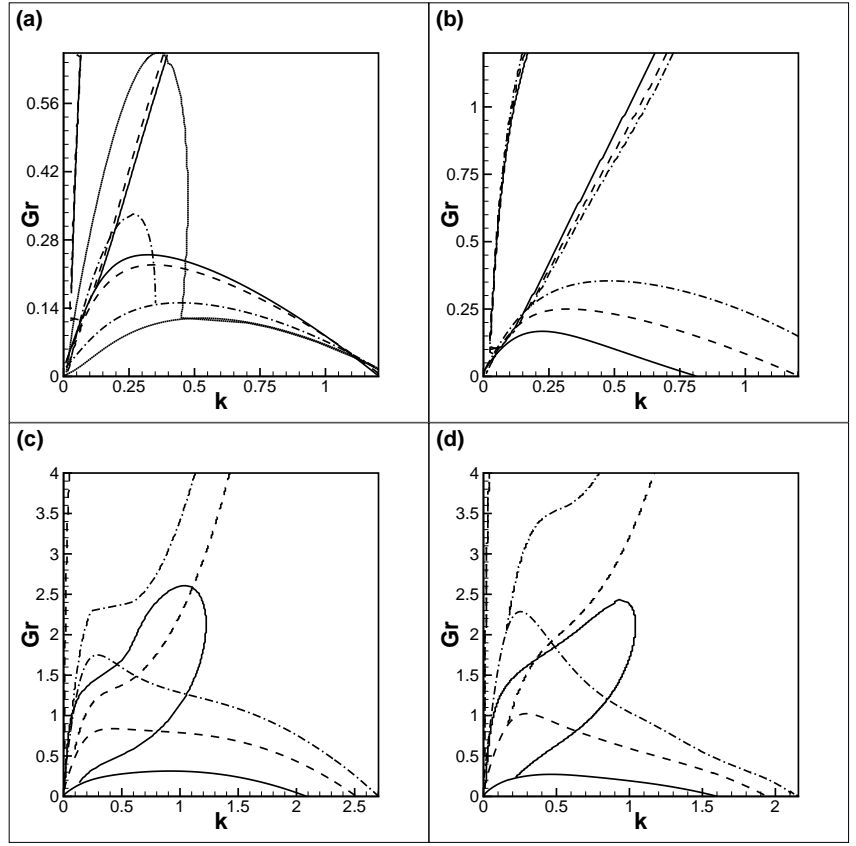

Fig. 12. The neutral curves are plotted for a miscible interface between two liquids with the heavier liquid underneath. The curves are obtained for $\delta_{u}=1, P e=100$, (a) $R e=25, \delta=0.3$, $C a=0.05, A=-0.5$ (solid lines), $A=-0.3$ (dashed lines), $A=0.3$ (dash-dot lines), and $A=0.5$ (dotted lines); (b) $R e=25, \delta=0.3, A=0.5, C a=0$ (solid lines), $C a=0.05$ (dashed lines), and $C a=0.1$ (dash-dot lines); (c,d) $R e=10$, $C a=0.05, \delta=0.05, U_{0}=1$ (solid lines), $U_{0}=2$ (dashed lines), and $U_{0}=3$ (dash-dot lines), and $A=-0.5$ (c) and $A=0.5$ (d).

some of the modes (with longer wavelengths) start growing faster in the presence of the flows, but the growth of short wave modes is slowed down. Diffusivity introduces additional mechanism of dissipation, and its action is similar to the influence of the viscous force. Capillarity reduces the growth rates, primarily affecting the development of the modes of shorter wavelengths. If the capillary forces are neglected, then the gradual decrease of the interface thickness can reveal the sharp-interface behaviour. If however the capillary terms are non-negligible, then the decrease in the interface thickness does not produce the convergence to any limiting curve. When $C a$ and $\delta$ were both simultaneously decreased at the same rate, the limiting behaviour was not revealed either.

The configuration with the heavier liquid placed underneath the lighter one is stable if no flow is imposed. The flow driven along the interface surface destabilises the interface by introducing the Kelvin-Helmholtz and Holmboe instabilities. The interface would remain stable at very high Grashof numbers, but at low Grashof numbers the flow makes the interface unstable. Usually, both the zones of the Kelvin-Helmholtz and Holmboe instabilities can be identified. The zone of the Kelvin-Helmholtz instability lies at lower Grashof numbers, and the zone of the Holmboe instability is attached to the zone of the KelvinHelmholtz instability and usually extends to significantly 


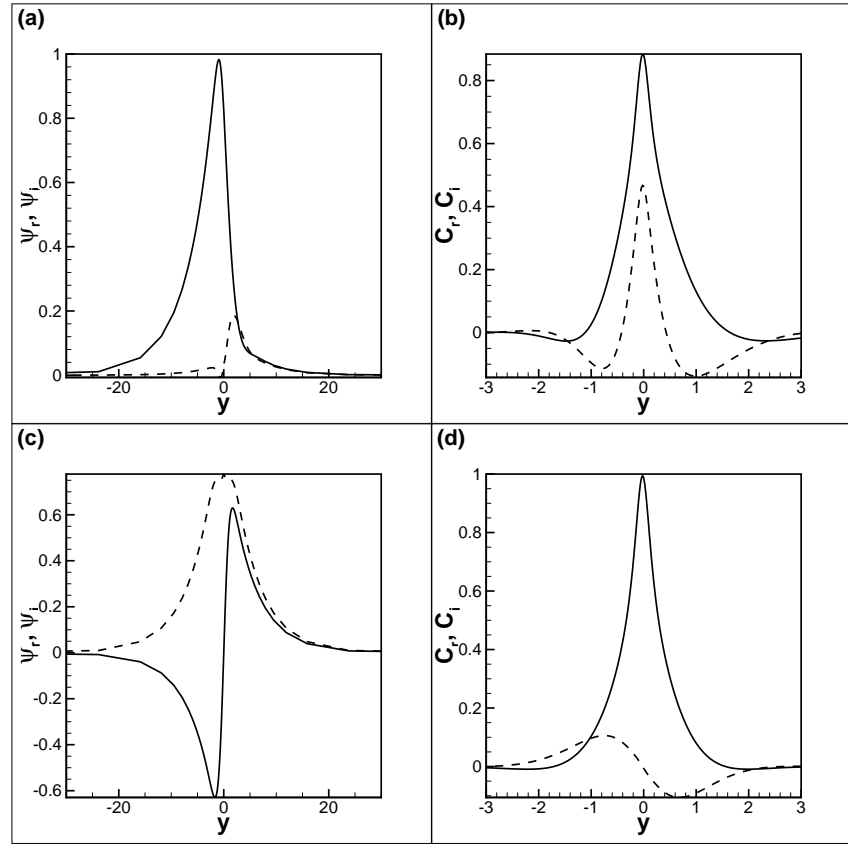

Fig. 13. The eigenfunctions, namely the profiles of streamfunction $(\mathbf{a}, \mathbf{c})$ and concentration $(\mathbf{b}, \mathbf{d})$ are shown for perturbations developing at a miscible interface that separates two liquids with the heavier liquid underneath. The functions are plotted for $k=0.2, P e=100, C a=0.05, \delta=0.1, R e=25, A=0.5$ and for $(\mathbf{a}, \mathbf{b}) G r=0.1$ (the range of the Kelvin-Helmholtz instability) and (c,d) $G r=1$ (the range of Holmboe instability). The solid lines depict the real parts, and the dashed lines show the imaginary parts. The eigenvalues are $\omega=(0,0.087)$ (for $\mathrm{a}, \mathrm{b})$ and $\omega=(0.283,0.011)$ (for $\mathrm{c}, \mathrm{d})$.

higher Grashof numbers. As expected, the zones of instability are significantly enlarged for the flows with higher amplitudes. Surprisingly, we found that interfacial diffusion also expands the zones of instability. The interface is also more unstable if its thickness is small. However, in the case of $A<0$, very thick interfaces, with thicknesses exceeding the equilibrium value $\delta_{0}$, become unconditionally unstable due to the thermodynamic instability. In addition, the instability zones are increased if the capillary effects are added. Viscosity however suppresses the instability reducing the range of unstable modes.

\section{Acknowledgements}

The work was supported by Russian Scientific Foundation (grant No. 14-21-00090).

\section{References}

1. A. Kheniene, A. Vorobev, Physical Review E 88, 022404 (2013)

2. T. Babadagli, Journal of Petroleum Science and Engineering 57, 221 (2007)

3. M. Mukhopadhyay, Natural extracts using supercritical carbon dioxide (CRC Press LLC, 2000)
4. E. B. Nauman, A. Nigam, Trans IChemE, Part A, Chemical Engineering Researchand Design 85, 612 (2007)

5. A. Vorobev, Current Opinion in Colloid and Interface Science 19, 300 (2014)

6. S. Chandrasekhar, Hydrodynamic and Hydromagnetic Stability (Oxford University Press, 1961)

7. P. Drazin, W. Reid, Hydrodynamic instability (Cambridge University Press, 1981)

8. P. N. Guzdar, P. Satyanarayana, J. D. Huba, et al., Geophysical Research Letters 9, 547 (1982)

9. W. Zhang, Z. Wu, D. Li, Physics of Plasmas 12, 042106 (2005)

10. B. J. Olson, J. Larsson, S. K. Lee, et al., Physis of Fluids 23, 114107 (2011)

11. L. F. Wang, W. H. Ye, Y. J. Li, Physics of Plasmas 17, $042103(2010)$

12. W. H. Ye, L. F. Wang, C. Xue, et al., Physics of Plasmas 18, 022704 (2011)

13. J. Holmboe, Geophys. Publ. 24, 67 (1962)

14. P. G. Drazin, Journal of Fluid Mechanics 4, 214 (1958)

15. S. A. Maslowe, R. E. Kelly, Journal of Fluid Mechanics 48, 405 (1971)

16. P. Hazel, Journal of Fluid Mechanics 51, 39 (1972)

17. L. N. Howard, S. A. Maslowe, Boundary-Layer Meteorology 4, 511 (1973)

18. P. G. Baines, H. Mitsudera, Journal of Fluid Mechanics 276, 327 (1994)

19. J. R. Carpenter, N. J. Balmforth, G. A. Lawrence, Physics of Fluids 22, 054104 (2010)

20. G. A. Lawrence, S. P. Haigh, Z. Zhu, Coastal and Estuarine Studies 54, 295 (1998)

21. S. P. Haigh, G. A. Lawrence, Physics of Fluids 11, 1459 (1999)

22. S. A. Thorpe, W. D. Smyth, L. Li, Journal of Fluid Mechanics 731, 461 (2013)

23. S. Alabduljalil, R. H. Rangel, Journal of Engineering Mathematics 54, 99 (2006)

24. R. Barros, W. Choi, Physics of Fluids 23, 124103 (2011)

25. S. A. Thorpe, Journal of Fluid Mechanics 32, 693 (1968)

26. S. A. Thorpe, Radio Science 4, 1327 (1969)

27. A. M. Hogg, G. N. Ivey, Journal of Fluid Mechanics 477, $339(2003)$

28. G. M. Corcos, F. S. Sherman, Journal of Fluid Mechanics 73, 241 (1976)

29. W. D. Smyth, Journal of Fluid Mechanics 497, 6798 (2003)

30. J. Lowengrub, L. Truskinovsky, Proc. R. Soc. Lond A 454, 2617 (1998)

31. A. Vorobev, Physical Review E 82, 056312 (2010)

32. L. D. Landau, E. M. Lifshitz, Course of Theoretical Physics. Volume 5. Statistical Physics (Elsevier. Butterworth Heinemann, 1980)

33. M. S. P. Stevar, A. Vorobev, Journal of Colloid and Interface Science 383, 184 (2012)

34. C. Pozrikidis, Journal of Fluid Mechanics 351, 139 (1997)

35. D. Bettinson, G. Rowlands, Physical Review E 54, 6102 (1996)

36. S. D. Conte, SIAM Review 8, 309 (1966)

37. L. M. Mack, Journal of Fluid Mechanics 73, 497 (1976)

38. A. Tumin, ASME J. Fluids Eng. 125, 428 (2003)

39. R. Betchov, A. Szewczyk, Physics of Fluids 6, 1391 (1963)

40. S. A. Maslowe, Hydrodynamic instabilities and the transition to turbulence, chap. Shear flow instabilities and transition, 181-228 (Springer Berlin Heidelberg, 1981) 
41. A. Vorobev, O. Zikanov, Journal of Fluid Mechanics 574, 131 (2007)

42. C.-C. P. Caulfield, Journal of Fluid Mechanics 258, 255 (1994) 